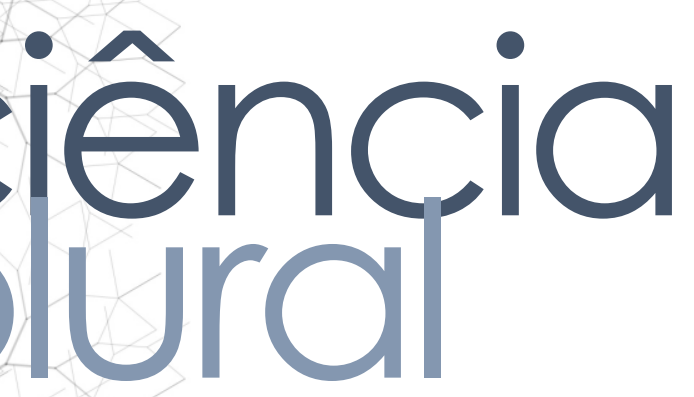

\title{
INVASÕES TERRITORIAIS POR PROCEDIMENTOS OBSTÉTRICOS EM UMA REGIÃO DE SAÚDE DO RIO GRANDE DO NORTE
}

Territorial Invasions in a Health Region of Rio Grande do Norte in Virtue of Obstetric Procedures

Invasiones Territoriales en una Región Sanitaria de Rio Grande do Norte en Virtud de Procedimientos Obstétricos

Gessica Rodrigues Cardoso • Sanitarista pela Universidade Federal do Rio Grande do Norte-UFRN・E-mail: gessica.cardosorn@gmail.com

Flávia Christiane de Azevedo Machado • Professora Adjunta do Departamento de Saúde Coletiva • Universidade Federal do Rio Grande do Norte • E-mail:

flavitamachado@yahoo.com.br

Deborah Jennifer de Paiva Lins • Sanitarista pela Universidade Federal do Rio Grande do Norte • E-mail: deborah.jennifer@hotmail.com

Maria Clara Pinheiro de Lima • Sanitarista pela Universidade Federal do Rio Grande do Norte E-mail: mclarapinheiro@live.com

Autora correspondente:

Gessica Rodrigues Cardoso • E-mail: gessica.cardosorn@gmail.com 


\section{RESUMO}

Introdução: No ponto de vista organizativo do Sistema Único de Saúde, a descentralização e a regionalização visam ampliar a resolutividade em nível local, reduzindo a desigualdade na assistência à saúde da população. Em meio às dificuldades de financiar os serviços dos três níveis de complexidades assistenciais em todos os municípios, opta-se pela formação de Regiões de Saúde. No Rio Grande do Norte, seus 167 municípios dividem-se em oito Regiões de Saúde. Objetivo: Identificar os procedimentos obstétricos e Estado que mais frequentemente ocasionou as invasões territoriais na assistência à saúde na VI região de saúde do Rio Grande do Norte em 2018. Metodologia: Estudo quantitativo, de caráter exploratório e descritivo realizado com dados secundários, referentes a 2018, captados no Departamento de Informática do Sistema Único de Saúde e banco de dados da programação pactuada e produção hospitalar cedido pela Secretaria Estadual de Saúde Pública do Rio Grande do Norte. Resultados: Indicaram a existência da invasão territorial na região de saúde, afirmando maior representatividade dessa invasão pelos Estados limítrofes. Conclusões: A invasão territorial se mostra presente na região de saúde e no Estado, despertando olhares sobre tal questão e suas implicações, potencializando novas inquietações.

Palavras-Chave: Sistema Único de Saúde, Migração Interna, Migração Humana, Saúde Pública, Obstetrícia, Regionalização.

\section{ABSTRACT}

Introduction: From the organizational point of view of the Unified Health System, decentralization and regionalization aim to expand resolvability at the local level, reducing inequality in health care for the population. In the midst of the difficulties of financing the services of the three levels of assistance complexities in all municipalities, the option is for the formation of Health Regions. In Rio Grande do Norte, its 167 municipalities are divided into eight Health Regions. Objective: Identify the obstetric and State procedures that most often caused territorial invasions in health care in the VI health region of Rio Grande do Norte in 2018. Methodology: Quantitative, exploratory and descriptive study conducted with secondary data, referring to 2018, captured in the Informatics Department of the Unified Health System and database of the agreed schedule and hospital production provided by the State Secretariat of Public Health of Rio Grande do Norte. Results: They indicated the existence of territorial invasion in the health region, affirming the greater representativeness of this invasion by neighboring states. Conclusions: The territorial invasion appears to be present in the health region and in the State, awakening views on this issue and its implications, potentiating new concerns.

eywords: Unified Health System, Internal Migration, Human Migration, Public lealth, Obstetrics, Regionalization. 


\section{RESUMEN}

Introducción: Desde el punto de vista organizativo del Sistema Único de Salud, la descentralización y la regionalización apuntan a ampliar la resolubilidad a nivel local, reduciendo la desigualdad en la atención de salud de la población. En medio de las dificultades para financiar los servicios de los tres niveles de complejidad asistencial en todos los municipios, la opción es por la formación de Regiones de Salud. En Rio Grande do Norte, sus 167 municipios se dividen en ocho Regiones de Salud. Objetivo: Identificar los procedimientos obstétricos y estatales que con mayor frecuencia causaron invasiones territoriales en la atención de la salud en la VI Región de Salud de Rio Grande do Norte en 2018. Metodología: Estudio cuantitativo, exploratorio y descriptivo realizado con datos secundarios, referidos a 2018, capturados en el Departamento de Informática del Sistema Único de Salud y base de datos del cronograma acordado y producción hospitalaria proporcionada por la Secretaría de Estado de Salud Pública de Rio Grande do Norte. Resultados: Señalaron la existencia de invasión territorial en la región de la salud, afirmando la mayor representatividad de esta invasión por parte de los estados vecinos. Conclusiones: La invasión territorial parece estar presente en la región de la salud y en el Estado, despertando visiones sobre este tema y sus implicaciones, potencializando nuevas preocupaciones.

Palabras clave: Sistema Único de Salud, Migración Interna, Migración Humana, Salud Pública, Obstetricia, Regionalización. 


\section{Introdução}

A Constituição da República Federativa do Brasil de 1988 legaliza a criação do Sistema Único de Saúde (SUS), assegurando a saúde como um direito social e de garantia do Estado. O SUS é composto por ações e serviços públicos de saúde integrantes de uma rede regionalizada e hierarquizada, direcionada pela descentralização, integralização e participação social. No entanto, a hierarquia não deve ser compreendida no sentido de nível de poder, mas sim da otimização dos recursos disponíveis de forma dialogada e negociada entre os entes federados ${ }^{1}$.

Não obstante, os serviços de saúde devem atuar de forma articulada como uma Rede de Atenção garantindo o fluxo de informações e pessoas. Quanto maior a possibilidade de acessar os diferentes pontos da Rede, mais fluída esta é e, potencialmente, permite a atenção integral ao indivíduo em conformidade as suas necessidades e em tempo oportuno².

Além da integralidade da atenção, o SUS tem por princípios doutrinários a universalidade de acesso em todos os níveis de complexidade da assistência à saúde; sem preconceitos e privilégio; a equidade. No ponto de vista organizativo, a descentralização político-administrativa configura-se na municipalização da gestão e na regionalização da organização dos serviços, com o intuito de ampliar a resolutividade em nível local ${ }^{3,4}$.

Neste sentido, os princípios vão ao encontro do objetivo finalístico da criação do SUS em alterar a situação de desigualdade na assistência à saúde da população, tornando obrigatório o atendimento público a qualquer cidadão, ofertando serviços na atenção primária, secundária e terciária ${ }^{5}$.

Em meio às dificuldades de financiar os serviços dos três níveis de complexidades assistenciais em todos os municípios, opta-se pela formação de Regiões de Saúde e, através de pactos no âmbito dessas regiões, torna-se possível a ferta da atenção integral. 
A Regionalização vem sendo discutida e normatizada por diferentes instrumentos ao longo da história do SUS. Neste sentido, para integrar os três entes federados e definir responsabilidades sanitárias têm sido instituídos instrumentos normativos do SUS para além das Leis Orgânicas e Normas Operacionais Básicas (NOBs) e Norma Operacional de Assistência à Saúde (NOAS). Assim, em 2006 instituiu-se o Pacto pela Saúde, dividido em três eixos estruturantes: Pacto pela Vida, Pacto em Defesa do SUS e Pacto de Gestão do SUS. O eixo Pacto de Gestão do SUS enfatiza a importância da territorialização da saúde como base na organização dos sistemas, apresentando a descentralização e a regionalização como ordenador para o fortalecimento da gestão compartilhada e solidária do SUS6 .

Em 2011 é publicado o Decreto $n^{\circ} 7.508$, que regulamenta a lei $n^{\circ} 8.080 / 90$ e aborda a organização do SUS e a articulação interfederativa, dentre outras providências. O decreto apresenta o conceito de Região de Saúde (RS), como: "espaço geográfico contínuo constituído por agrupamentos de Municípios limítrofes, delimitado a partir de identidades culturais, econômicas e sociais e de redes de comunicação e infraestrutura de transportes compartilhados, com a finalidade de integrar a organização, o planejamento e a execução de ações e serviços de saúde"7.

Ainda segundo o Decreto, para ser instituída, a Região de Saúde deve conter, no mínimo, ações e serviços de atenção primária; urgência e emergência; atenção psicossocial; atenção ambulatorial especializada e hospitalar e vigilância em saúde. Por sua vez, os entes federativos devem definir em relação às Regiões de Saúde os seus limites geográficos; a população usuária das ações e serviços; o rol de ações e serviços que serão ofertados; as respectivas responsabilidades, critérios de acessibilidade e escala para conformação dos serviços ${ }^{7}$.

As iniciativas necessárias à operacionalização das diretrizes e objetivos stabelecidos para a região e que contribuirão para o alcance das metas são definidas Programação Geral das Ações e Serviços de Saúde (PGASS). A PGASS permite, a artir de estimativas de necessidades em saúde, apontar os investimentos cessários, antes de promover a alocação dos recursos de custeio em conformidade fluxos assistenciais para ações de promoção e assistência à saúde, de vigilância 


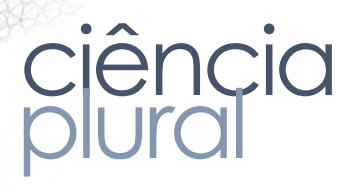

(sanitária, epidemiológica e ambiental) e de assistência farmacêutica. Portanto, ao dimensionar as ações de serviços de saúde pelo percentual de alcance do ano vigente na Programação Anual de Saúde, pactuam-se os fluxos assistenciais, mediante a definição de municípios executores e encaminhadores, para cada item de programação $^{8}$.

Esta pactuação pode ocorrer por abrangência ou por referência. Por referência acontece quando os quantitativos programados são alocados no(s) município(s) executor(es) pelos municípios encaminhadores, em um processo de pactuação no âmbito da Comissão Intergestora Regional (CIR). Por abrangência, acontece quando os quantitativos programados são alocados no(s) município(s) executor(es) e em um processo de pactuação, no âmbito da Comissão Intergestora Bipartite (CIB), são definidos os municípios a serem abrangidos por executor e respectivas metas. $\mathrm{O}$ cálculo do impacto financeiro será realizado a partir da quantificação das ações e serviços de saúde e dos fluxos estabelecidos na pactuação ${ }^{8}$.

Desta forma, é possível inferir que a não conformidade das pactuações realizadas tem implicações na exequibilidade e, por conseguinte, na garantia da atenção integral pretendida pelo SUS. Na perspectiva da não conformidade as pactuações, vem se destacando a discussão sobre as migrações. As migrações consistem na mobilização da população de um local para outro, modificando ou interferindo nas características de uma região administrativa. No tocante a Região de Saúde, coloca-se a questão da migração temporária, onde as pessoas se deslocam em busca de assistência à saúde, configurando a invasão territorial no Sistema Único de Saúde ${ }^{9}$. Um exemplo prático dessa migração temporária, é na busca pontual na assistência para partos e/ou intercorrências gestacionais, exigindo organização e agilidade dos serviços de saúde. Sendo assim, a fase gestacional requer atenção dos gestores do SUS no planejamento e pactuações das ações e serviços disponíveis para uas populações, de forma a assegurar o cuidado necessário à mulher e a criança ${ }^{10}$.

Organizar e operacionalizar as ações e serviços assistenciais no Sistema Único Saúde de forma regionalizada são grandes desafios, sendo a ausência de rmações que subsidiem um planejamento coerente com a dinâmica local um fator 
de significativa fragilidade, comprometendo a efetivação dos princípios do SUS e, por conseguinte, a viabilidade de uma atenção à saúde integral.

O Estado do Rio Grande do Norte (RN) tem uma área territorial de 52.809,602 km² e população estimada em 2019 de 3.506.853 habitantes, tendo por limites o Oceano Atlântico a norte e a leste, a Paraíba (PB) ao sul e o Ceará (CE) a oeste ${ }^{11}$. O Estado divide-se em 167 municípios e 08 regiões de saúde, denominadas: I RS - São José de Mipibu (com 27 municípios), II RS - Mossoró (com 14 municípios), III RS João Câmara (com 26 municípios), IV RS - Caicó (com 25 municípios), V RS - Santa Cruz (com 21 municípios), VI RS - Pau dos Ferros (com 37 municípios), VII RS Metropolitana (com 05 municípios) e VIII RS - Assu (com 12 municípios) ${ }^{12}$.

Neste contexto, o estudo buscará conhecer os procedimentos obstétricos e os Estados que ocasionam as invasões territoriais da assistência à saúde na VI região de saúde do Rio Grande do Norte no ano de 2018.

\section{Metodologia}

Trata-se de um estudo de abordagem quantitativa e de caráter exploratório e descritivo realizado com dados secundários captados no Departamento de Informática do Sistema Único de Saúde - DATASUS e analisados usando o TABWIN. Busca-se captar informações sobre os procedimentos obstétricos relacionados ao fenômeno das invasões territoriais na VI região de saúde - Pau dos Ferros do Rio Grande do Norte, referente ao ano de 2018. No Sistema Único de Saúde os procedimentos, ações e serviços de saúde vinculam-se aos usuários e estabelecimentos de saúde, através do Cartão Nacional do SUS (Cartão SUS).

As invasões territoriais, neste estudo, são identificadas a partir da estratificação dos dados referentes a Unidade da Federação de Residência versus CIR de Internação. O Estado de residência das usuárias dos SUS foi o fator determinante para a caracterização da "invasão territorial". Ressalte-se que não há um marco eórico para tal definição, uma vez que esta discussão é muito recente.

O TABWIN, disponível para download de forma gratuita, foi criado pelo TASUS para facilitar o trabalho de tabulação e o tratamento de dados. Dentre 
suas funcionalidades permite a realização de operações aritméticas e estatísticas nos dados da tabela gerada ou importada pelo Tabwin. Os dados e informações viabilizadas são um subsídio para o planejamento e programação de serviços, bem como a avaliação do impacto de intervenções nas condições de saúde ${ }^{13}$.

A VI Região de Saúde - Pau dos Ferros possuiu no ano de 2018 uma população estimada em 253.894 habitantes e densidade demográfica: 5.23 hab/km211. É composta por 37 municípios, descriminados: Água Nova, Alexandria, Almino Afonso, Antônio Martins, Coronel João Pessoa, Doutor Severiano, Encanto, Francisco Dantas, Frutuoso Gomes, Itaú, João Dias, José da Penha, Lucrécia, Luís Gomes, Major Sales, Marcelino Vieira, Martins, Olho D'água dos Borges, Paraná, Pau dos Ferros, Patú, Pilões, Portalegre, Rafael Fernandes, Rafael Godeiro, Riacho da Cruz, Riacho de Santana, Rodolfo Fernandes, São Francisco do Oeste, São Miguel, Serrinha dos Pintos, Severiano Melo, Taboleiro Grande, Tenente Ananias, Umarizal, Venha-Ver e Viçosa ${ }^{15}$.

Considerou-se como cenário do estudo a VI Região de Saúde - Pau dos Ferros, em virtude de ser a única região de saúde do Rio Grande do Norte com maior área territorial limítrofe em dois Estados: Paraíba e Ceará, e possuir o maior número de municípios em sua composição em relação as demais regiões de saúde.

Considerou-se o exercício de 2018 por apresentar produção fechada, considerando a temporalidade, junto aos sistemas de informações em saúde: Sistema de Informações Hospitalares (SIH) e Sistema de Informações sobre Nascidos Vivos (SINASC) do Ministério da Saúde. E foram excluídas as demais regiões de saúde do RN por apresentarem fronteira com o Oceano Atlântico e fronteiras com apenas um Estado.

O período do estudo contempla todas as competências de 2018, foram utilizados dados secundários, a coleta de dados ocorreu no período de Outubro a Jovembro de 2019, nos sistemas SIH, SINASC, com auxílio do TABWIN, TABNET e NES (Cadastro Nacional de Estabelecimentos de Saúde) para exploração e zamento dos dados, os quais foram analisados segundo suas frequências plutas e relativas. Os dados referentes a procedimentos obstétricos, englobam 
assim, partos normais (vaginais), cesáreos, curetagens pós-aborto e outras intervenções obstétricas.

E também foram utilizados dados disponibilizados pela SESAP/RN, cujo banco de dados é fruto do cruzamento de informações do Sistema de Programação Pactuada e Integrada (SISPPI) estadual e SIH, unificando informações da programação pactuada e produção hospitalar, cabe ressaltar que o banco de dados dispunha apenas de valores em reais $(\mathrm{R} \$)$ da assistência em saúde correspondente aos municípios da região de saúde de interesse, inexistindo qualquer relação direta ou indireta a seres humanos, não sendo necessário submeter ao Comitê de Ética. De posse dos dados, elaboraram-se tabelas e os gráficos com o auxílio do software Excel 2013.

\section{Resultados e Discussão}

A apresentação dos resultados e a discussão foram organizadas em torno dos objetivos norteadores e, desta forma, espera-se objetividade e clareza na exposição dos mesmos.

Identificou-se que no ano de 2018, a VI Região de Saúde - Pau dos Ferros realizou um total de 3.620 (100\%) procedimentos obstétricos, sendo que, 3.103 (86\%) correspondem às assistências aos residentes do próprio Rio Grande do Norte e 517 (14\%) correspondem a invasão territorial de outros Estados.

Os dados mostram que existiu a assistência obstétrica para usuárias de outros Estados dentro da VI RS, representando um valor de invasão territorial de 517 (14\%) em 2018. Os outros 3.103 (86\%) correspondem a assistência aos residentes no RN. Os achados demonstram consonância aos princípios doutrinários do SUS, que expressam uma lógica positiva, apesar das dificuldades inerentes a utilização dos serviços de saúde 5 .

No que se refere à identificação dos procedimentos obstétricos, apareceram maior frequência, em ordem decrescente: 500 (96,71\%) ocorrências de parto sariano, $12(2,32 \%)$ de parto normal (vaginal), 4 (0,77\%) de curetagem pósrtamento/puerperal e $1(0,19 \%)$ de tratamento de intercorrências clínicas na idez. 
O parto cesariano destaca-se como o principal procedimento obstétrico motivador da invasão territorial na região de saúde de Pau dos Ferros. O resultado de 500 ocorrências $(96,71 \%)$ é coerente aos elevados índices de cesárea a nível de Brasil. Em 2010, no Brasil tiveram cerca de 1.496.034 (52\%) de partos cirúrgicos, bem acima do estabelecido pela Organização Mundial de Saúde (OMS), que considera um percentual em torno de 15\% como aceitável. Este percentual teve por consequência, queixas sobre os efeitos das cesarianas e sua inadequada realização. Além disso, há aumento da possibilidade de complicações que podem levar a morte tanto da mulher quando da criança. Assim, configura-se uma violência contra mulher, uma vez que fere a dignidade e respeito ${ }^{16}$. Desde 2015 que o Rio Grande do Norte possui um Protocolo de Assistência Materno Infantil, para auxiliar as equipes de saúde quanto as práticas adequadas na assistência, vez que os partos e quaisquer intercorrências gestacionais requerem cuidados que reduzam os riscos à mulher e criança. $\mathrm{O}$ parto cesariano é um procedimento cirúrgico de alto custo e em sua maioria é eletivo, ou seja, com agendamento. E o elevado percentual de partos cesarianos encontrados na VI RS do RN alerta sobre as condutas adotadas; a quebra da qualidade do cuidado; inutilização dos instrumentos de planejamento em saúde pelos gestores; comportamento das pactuações e atuação do controle social, que tendem a subsidiar análises futuras ${ }^{17}$.

Em relação às invasões por Unidade da Federação (UF), foi registrada a presença de 12 Estados: Goiás (GO), Mato Grosso (MT), Mato Grosso do Sul (MS), Rondônia (RO), Santa Catarina (SC), São Paulo (SP), Rio de Janeiro (RJ), Ceará (CE), Paraíba (PB), Pernambuco (PE), Alagoas (AL) e Bahia (BA). Quando se analisa o Estado que mais invadiu em busca de procedimentos obstétricos na VI região de saúde, observa-se que a Paraíba realizou 354 procedimentos $(68,47 \%)$, seguido do Ceará, com 139 procedimentos (26,89\%), conforme disposto na Tabela 1. 


\section{ciência
purál}

Tabela 1 - Unidades federativas que invadiram a VI RS em procedimentos obstétricos, 2018. Natal/RN, 2019.

\begin{tabular}{ccc|}
$\begin{array}{c}\text { Unidade Federativa } \\
\text { (UF) }\end{array}$ & $\begin{array}{c}\text { Total de Proc. } \\
\text { Obstétricos } \\
\text { (absoluto) }\end{array}$ & $\begin{array}{c}\text { Percentual de } \\
\text { Proc. Obstétricos } \\
\text { (\%) }\end{array}$ \\
\hline Rondônia & 1 & $0,19 \%$ \\
Ceará & 139 & $26,89 \%$ \\
Paraíba & 354 & $68,47 \%$ \\
Pernambuco & 3 & $0,58 \%$ \\
Alagoas & 1 & $0,19 \%$ \\
Bahia & 1 & $0,19 \%$ \\
Rio de Janeiro & 1 & $0,19 \%$ \\
São Paulo & 7 & $1,35 \%$ \\
Santa Catarina & 6 & $1,16 \%$ \\
Mato Grosso do Sul & 1 & $0,19 \%$ \\
Mato Grosso & 1 & $0,19 \%$ \\
Goiás & 2 & $0,39 \%$ \\
TOTAL & $\mathbf{5 1 7}$ & $\mathbf{1 0 0} \%$
\end{tabular}

Fonte: TABWIN/DATASUS, 2019.

A VI RS Pau dos Ferros possui 37 municípios em sua composição, desses, 15 são limítrofes a outros Estados: 07 limítrofes com o Ceará e 08 com a Paraíba. Ressalta-se ainda, que o município de Venha-Ver possui fronteiras territoriais tanto com a Paraíba quanto com o Ceará.

Constatou-se que o Estado da Paraíba foi o que apresentou maior frequência de invasão, seguido do Estado do Ceará, reafirmando a tendência de migração temporária na busca por serviços assistenciais em saúde ${ }^{9}$. Verificou-se também a assistência em procedimentos obstétricos para usuários de Estados fora do Nordeste, como foi o caso dos Estados: Goiás, Mato Grosso, Mato Grosso do Sul, Rondônia, Santa Catarina, São Paulo e Rio de Janeiro. Destacando que Santa Catarina é o Estado com maior distância em relação ao Rio Grande do Norte, com 3.566,4 km. Cabe ressaltar que, o Rio Grande do Norte recebe um grande número de turistas ao longo ano, pelo forte turismo no Estado, o que favorece hipoteticamente o surgimento sses na estratificação de dados como um todo. Inclusive, a disposição de rodovias stradas que facilitam a conexão com os Estados vizinhos. Como é o caso, por 
exemplo, das rodovias: BR-101 (que liga o Rio Grande do Norte à Paraíba) e BR-304 (que liga o Rio Grande do Norte ao Ceará) ${ }^{18}$. Além de outros motivos possíveis, que caracterizam as invasões territoriais na VI região de saúde, podem estar relacionados a desatualização cadastral do Cartão SUS, vez que os procedimentos realizados no âmbito do SUS vinculam-se as usuárias através dele. Para a identificação das invasões territoriais neste estudo, considerou-se a UF de residência, logo, a desatualização desse item no cartão SUS, compromete a fidedignidade dos dados nos sistemas de informações em saúde, introduzindo um viés de erro nas análises assistenciais da região.

Em 2018, a região de saúde possuía 418 (9\%) estabelecimentos de saúde cadastrados no CNES. Com 77 (39\%) leitos hospitalares e 121 (61\%) leitos de internações obstétricos. Em relação ao número de profissionais de saúde cadastrados, foram identificados $07(0,2 \%)$, divididos entre: enfermeiro(a) obstétrico, Ginecoobstetra e Médico Ginecologista Obstetra14. Cabe ressaltar quanto ao número de profissionais, a possibilidade de existir um número maior atuando na assistência obstétrica, porém, podem estar cadastrados em outras especialidades assistenciais, a exemplo, de um Médico Obstetra que pode estar cadastrado como Clínico Geral.

Destaca-se ainda, a participação dos Gestores em Saúde nos espaços de negociações, desconsiderando, muitas vezes, a análise da situação de saúde nas tomadas de decisões e/ou articulações das pactuações nos colegiados. Fragilizando as articulações na região e os processos de regionalização ${ }^{19}$.

Dos $37(100 \%)$ municípios que compõem a VI região de saúde, apenas 07 (19\%) proporcionaram uma assistência obstétrica para usuários caracterizados na invasão territorial, conforme representa o Mapa 1. No caso, Alexandria foi o município que mais assistiu, realizando 371 (72\%) procedimentos obstétricos de um total de 517 (100\%) realizados na região de saúde. Foram evidenciadas assistências pontuais em Almino Afonso, Patu, Frutuoso Gomes e Rodolfo Fernandes. 
Mapa 1 - Municípios que assistiram as invasões territoriais em procedimentos obstétricos na VI RS do Rio Grande do Norte, 2018. Natal/RN, 2019.

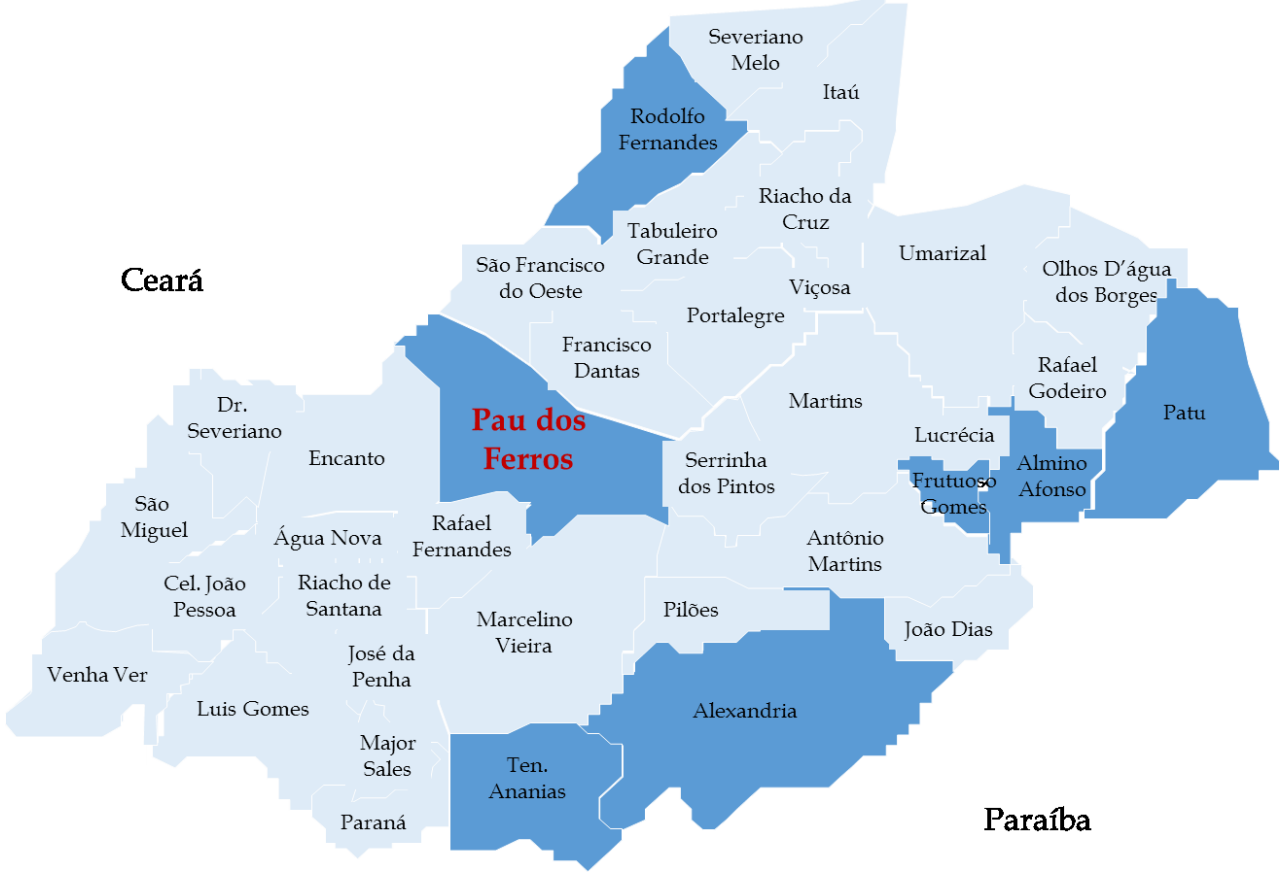

Fonte: TABWIN/DATASUS, 2019.

Após a análise dos municípios da VI região de saúde que assistiram as invasões territoriais, observou-se que apenas $07(19 \%)$ municípios realizaram a cobertura assistencial em procedimentos obstétricos, sendo eles: Pau dos Ferros (cidade polo e referência da região de saúde), Tenente Ananias, Rodolfo Fernandes, Patu, Frutuoso Gomes, Almino Afonso e Alexandria. Um aspecto importante a ser observado, é que a cidade polo da VI RS assistiu a 64 (13\%) das invasões territoriais, em parto cesariano, por sua vez, a cidade de Alexandria assistiu a 368 (74\%). Pode-se associar tal efeito, por Alexandria possuir limites territoriais com três municípios da Paraíba: Santa Cruz, Bom Sucesso e Brejo dos Santos ${ }^{11}$, mas também desperta a indagação dos motivos que fazem essas mulheres irem para Alexandria nessa proporção. Nessa perspectiva as invasões territoriais apresentaram impacto financeiro na região de saúde de $\mathrm{R} \$ 330.031,16$ (trezentos e trinta mil, trinta e um eais e dezesseis centavos), em Pau dos Ferros município de referência da região vemos um impacto de $\mathrm{R} \$ 45.364,96$ (quarenta e cinco mil, trezentos e sessenta e tro reais e noventa e seis centavos) e Alexandria diante da representatividade da 
assistência prestada obteve $\mathrm{R}$ \$242.049,48 (duzentos e quarenta e dois mil, quarenta e nove reais e quarenta e oito centavos). Os valores correspondem a apenas uma região de saúde do Estado do Rio Grande do Norte, cabendo a reflexão do impacto que temos a nível estadual ao considerarmos as 08 regiões de saúde e principalmente, nas estratégias adotadas diante desse cenário.

O estudo encontrou como limitações a ausência de artigos publicados ou outros documentos que pudessem servir como subsídio para discussão dos achados no tocante às migrações relacionadas à temática das "invasões territoriais no SUS". No tocante a regionalização no RN, também há escassez de informações atualizadas no site da Secretaria Estadual de Saúde Pública. Apesar das limitações apontadas, essas não estão relacionadas à fragilidade do método ou a não relevância do tema sob estudo.

As "invasões territoriais" são uma discussão recente, não havendo publicações sobre o tema. Isto inviabiliza a comparação de resultados com outros estudos. De fato, as reflexões aqui realizadas foram possíveis em virtude da inserção da autora no cotidiano dos serviços.

As discussões relacionadas a logística de acesso são muito relevantes. As "invasões territoriais" partem de um pressuposto de uma fragilidade no acesso. Em se tratando de um sistema de saúde universal e de equidade na atenção, é deveras importante aprofundar os estudos sobre este fenômeno.

\section{Conclusões}

No tocante aos principais achados, em 2018, houve um percentual de invasão territorial de $14 \%$ na VI Região de Saúde do RN e o parto cesariano destacou-se como o principal procedimento obstétrico motivador da invasão territorial. A Paraíba e o Ceará foram os estados mais frequentes quanto à invasão territorial.

O estudo em questão viabiliza a oportunidade de ampliar as discussões sobre fator da gestão em saúde pouco explorado, as invasões territoriais na saúde. redita-se que este estudo, por seu caráter exploratório pode impulsionar novos 
estudos e contribuir nos processos de planejamento da VI região de saúde, bem como da própria SESAP/RN, ultrapassando as perspectivas acadêmicas.

\section{Referências}

1. Vilarins GCM, Shimizu HE, Gutierrez MMU. A regulação em saúde: aspectos conceituais e operacionais. Saúde em Debate, 2012; 36 (95):640-647.

2. Chueiri PS; Harzheim E; Takeda SMP. Coordenação do cuidado e ordenação nas redes de atenção pela Atenção Primária à Saúde - uma proposta de itens para avaliação destes atributos. Revista Brasileira de Medicina de Família e Comunidade, 2017; 12(39):1-18.

3. Viacava F, Oliveira RAD, Carvalho CC, Laguardia J, Bellido JG. SUS: oferta, acesso e utilização de serviços de saúde nos últimos 30 anos. Ciênc. Saúde Coletiva [online], 2018; 23(6): 1751-1762. [acesso em 07 dez 2019]. Disponível em: https:/ / www.scielo.br/scielo.php?script=sci_arttext\&pid=S141381232018000601751\&lng=pt.

4. Vasconcelos CM, Pasche D. O Sistema Único de Saúde. In: Campos GWS, Minayo MCS, Akerman M, Junior MD, Carvalho YM, (org). Tratado de Saúde Coletiva. São Paulo: Hucitec; Rio de Janeiro: Editora Fiocruz, 2006: 531-562.

5. Pontes APM, Oliveira DC, Gomes AMT. Os princípios do Sistema Único de Saúde estudados a partir da análise de similitude. Rev. Latino-Am. Enfermagem. 2014; 22(1).

6. Brasil. Ministério da Saúde (MS). Secretaria Executiva. Departamento de Apoio à Descentralização. Coordenação-Geral de Apoio à Gestão Descentralizada. Diretrizes operacionais dos Pactos pela Vida, em Defesa do SUS e de Gestão. Brasília: MS, 2006;76.

7. Brasil. Ministério da Saúde (MS). Decreto no 7.508: Regulamenta a Lei ${ }^{0} 8.080$, de 19 de setembro de 1990, para dispor sobre a organização do Sistema Único de Saúde SUS, o planejamento da saúde, a assistência à saúde e a articulação interfederativa, e dá outras providências. Diário Oficial da União 2011; 28 jun.

8. Brasil. Ministério da Saúde (MS). Secretaria de Atenção à Saúde. Departamento de Regulação, Avaliação e Controle de Sistemas Critérios e Parâmetros para o Planejamento e Programação de Ações e Serviços de Saúde no âmbito do Sistema Unico de Saúde. Brasília: MS 2015.

Ianni AMZ, Monteiro PHN, Alves OSF, Morais MLS, Barboza R. Metrópole e ião: dilemas da pactuação da saúde. O caso da Região Metropolitana da Baixada tista, São Paulo, Brasil. Cad Saúde Pública 2012; 28(5):925-934. 
10. Brasil. Ministério da Saúde (MS). Portaria $n^{0}$ 1459, de 24 de junho de 2011. Institui, no âmbito do Sistema Único de Saúde - SUS - a Rede Cegonha. Diário Oficial da União 2011; 24 jun.

11. IBGE - Instituto Brasileiro de Geografia e Estatística (IBGE). Estimativas da população, 2019. [acesso em 06 dez 2019]. Disponível em:

https:/ / www.ibge.gov.br/cidades-e-estados/rn.html.

12. Rio Grande do Norte (RN). Secretaria de Estado da Saúde Pública (SESAP). Coordenadoria de Planejamento e Controle de Serviços de Saúde. Plano Estadual de Saúde 2016-2019. Rio Grande do Norte; 2016. [acesso 01 dez 2019] Disponível em: http://www.adcon.rn.gov.br/ACERVO/sesap/DOC/DOC000000000241412.PDF.

13. Brasil. Ministério da Saúde (MS). Departamento de Informática do SUS DATASUS (TABWIN) [acesso em 06 dez 2019] Disponível em: http:/ / www2.datasus.gov.br/DATASUS/index.php?area=060805\&item=1.

14. Brasil. Ministério da Saúde (MS). Cadastro Nacional de Estabelecimentos de Saúde (CNES). [acesso em 01 dez 2019] Disponível em:

http:/ / www2.datasus.gov.br/DATASUS/index.php?area=0204.

15. Brasil. Ministério da Saúde (MS). Informações de Saúde (TABNET). [acesso em 01 dez 2019] Disponível em:

http:/ / www2.datasus.gov.br/DATASUS/index.php?area=02.

16. Pereira JS; Silva JCO; Borges NA; Ribeiro MMG; Auarek LJ; Souza JHVK. Violência Obstétrica: Ofensa à Dignidade Humana. Belo Horizonte. 2019;15(1):103108.

17. Rio Grande do Norte (RN). Secretaria de Estado da Saúde Pública (SESAP). Protocolo de assistência materno infantil do Estado do Rio Grande do Norte. Rio Grande do Norte; 2015. [acesso 01 dez 2019] Disponível em: http:/ / www.rnsustentavel.rn.gov.br/smiv3/site/conteudos/midias/917cdb466b65c eda7e06a4d28599a448.pdf.

18. Federação das Indústrias do Estado do Rio Grande do Norte (FIERN). Rio Grande do Norte; 2019. [acesso $01 \mathrm{dez} 2019]$ Disponível em:

https://www.fiern.org.br/rodovias/.

19. Teston LM; Mendes A; Carnut L; Louvison MCP. Desafios políticos e operacionais na percepção de gestores sobre a regionalização em saúde no Acre; 2019. Rio de Janeiro, 43(121):314-328. 